\title{
Feasibility Investigation of Developing a Revised MEWS Score for Cardiovascular Specialty
}

\author{
Yanling Zhang, Haiyan Zhuo, Huaili Bi, Juxian Wu* \\ Department of Cardionlogy, The People's Hospital of Zhuhai, Zhuhai, China \\ Email address: \\ 421235801@qq.com (Juxian Wu) \\ ${ }^{*}$ Corresponding author
}

\section{To cite this article:}

Yanling Zhang, Haiyan Zhuo, Huaili Bi, Juxian Wu. Feasibility Investigation of Developing a Revised MEWS Score for Cardiovascular Specialty. American Journal of Nursing Science. Vol. 10, No. 3, 2021, pp. 169-173. doi: 10.11648/j.ajns.20211003.16

Received: May 17,2021; Accepted: May 26, 2021; Published: June 10, 2021

\begin{abstract}
Objective: Cardiovascular disease is a series of diseases with high morbidity, high fatality rate, rapid changes in condition, and it is highly prone to emergencies and severe illnesses, and it is the primary disease that leads to death of patients. The modified early warning score (MEWS) is lacks specialized indicators and can't personalize risk prediction for cardiovascular patients. Explore the establishment of a scoring system suitable for evaluating the condition of inpatients in the Department of Cardiovascular Medicine to provide a reference basis for accurate assessment and adequate early warning of the patient's disease, and for targeted nursing plans. Methods: The questionnaire was designed through literature analysis, brainstorming, and expert interviews, and a questionnaire survey was conducted for nursing staff in the Department of Cardiology. Analyze the survey results through SSPS software. Results: According to the survey analysis, $73.9 \%$ of the experts surveyed considered it necessary or very necessary to establish a modified early warning score in cardiovascular medicine (MEWS); Approx. Chi-square value of the Bartlett sphericity test was 3760.769 (with 28 degrees of freedom), which reached a significant level $(\mathrm{P}<0.000)$, indicating that the reliability and validity of the scale were high. The corrected MEWS score scale was composed of 8 observation indexes, including systolic blood pressure, diastolic blood pressure, respiration, heart rate, oxygen saturation, arrhythmia, consciousness, and chest pain. Conclusion: Cardiovascular medical nursing staff had a high degree of approval for establishing the corrected MEWS score; The constructed MEWS score for cardiovascular internal disease correction is feasible, objective and practical to a certain extent, and can provide a basis for further exploration of practical application.
\end{abstract}

Keywords: Cardiovascular Disease, Corrected Early Warning Score, Feasibility Investigation, Questionnaire, Revised Score

\section{Introduction}

Cardiovascular disease is a series of diseases with high morbidity, high fatality rate, rapid changes in condition, and it is highly prone to emergencies and severe illnesses, and it is the primary disease that leads to death of patients [1]. Early and accurate identification of the patient's potential death and the risk of adverse events, and quantitative assessment of the severity of the disease are of great significance for guiding clinical work [2]. The modified early warning score (MEWS) is a tool developed by British experts to evaluate the conditions of patients with unclear condition at the time of emergency or admission, and to carry out risk classification to achieve the purpose of pre-check and triage [3]. Although it has been widely used for clinical emergency assessment [4-6], it lacks specialized indicators and can't personalize risk prediction for cardiovascular patients [7-8].
The purpose of this study is to investigate the current application status of MEWS in the Department of Cardiovascular Medicine, and to correct the MEWS based on the characteristics of cardiovascular diseases, so as to provide a scientific method for the objective assessment and timely warning of the trend of changes in the condition of inpatients in the Department of Cardiovascular Medicine.

\section{Materials and Methods}

\subsection{Research Team and Research Steps}

The research team consisted of seven cardiovascular internal nurses, including two assistant chief nurses, one chief nurse, and four nurses. The main research steps include literature review, determination of evaluation indicators; consultation of expert 
opinions, selection of selected indicators; preparation and distribution of questionnaires; recovery of questionnaires and statistical analysis; writing relevant research reports on the risk early warning evaluation indicator system.

\subsection{Research Object}

The convenience sampling was used to conduct a questionnaire survey of cardiovascular medical nursing staff across the country, in June 2019. A total of 220 questionnaires were distributed in this survey, and 207 were returned. The effective response rate of the questionnaires was $94.1 \%$.

\subsection{Cardiovascular Disease Correction MEWS Questionnaire}

\subsubsection{Research Methods}

Through the brainstorming method, the methods of literature review, expert consultation method, on the basis of the traditional MEWS score of heart rate and systolic blood pressure, breathing rate, body temperature and five average physiological indexes based on modified, modify the result is the temperature to be deleted, diastolic blood pressure, chest pain, arrhythmia, and blood oxygen saturation index is the net increase of four observations.

\subsubsection{Corrected Questionnaire}

(1) General Situation Questionnaire: the contents include the hospital level, professional title, educational background, working years of the respondents, and the application status of MEWS scoring in the department where the respondents worked;

(2) Questionnaire scoring criteria: selecting the "no need" option was counted as 1 point, and so on, the score was positively correlated with the importance of indicators.

\subsection{Statistical Methods}

Statistical analysis was performed using SPSS 22.0 statistical software package.

The description of the counting data is carried out by frequency and percentage (\%). The internal consistency reliability of the scale was evaluated by Cronbach's $\alpha$ coefficient; The construct validity of the scale is evaluated by KMO value and Bartlett's sphere test. $P<0.05$ indicates statistical significance. The validity of the expert consultation questionnaire is judged based on the missing rate of items. If the contents filled in are missing $>20 \%$, experts need to be contacted, and the second round of questionnaires will be issued to the experts again. If the missing contents reach $20 \%$ again, the questionnaire will be regarded as invalid.

\section{Result}

\subsection{The General Information}

The descriptive statistics of the demographic data of the survey subjects are shown in Table 1 . The 107 experts who accepted the survey, and filled in the questionnaire came from seven provinces, autonomous region, and municipalities directly under the central government, which had good universality, and representativeness. $82.1 \%$ of the experts came from the tertiary first-class tertiary, $60.3 \%$ of the experts with intermediate professional titles or above, $89.4 \%$ of the experts with bachelor's degree or above, and $95.1 \%$ of the experts with more than five years of working experience in cardiovascular medicine. The respondents of this survey have profound theoretical literacy and rich practical experience, which means that the study has a high authority.

Table 1. Descriptive statistics of demographic data of survey respondents ( $n=207$ ).

\begin{tabular}{|c|c|c|c|c|c|c|c|}
\hline Hospital Level & n\% & Job Title & $n \%$ & Education & n\% & Working Years & n\% \\
\hline Level 3 Class A & 82.1 & Senior title & 4.8 & Master & 2.4 & $15 \sim 20$ & 33.8 \\
\hline Level 3 Class B & 17.9 & Deputy senior title & 7.2 & Undergraduate & 87.0 & $10 \sim 14$ & 48.3 \\
\hline 1 & 1 & Intermediate title & 48.3 & Specialist & 10.6 & $5 \sim 9$ & 13.0 \\
\hline 1 & I & Junior title & 39.7 & 1 & 1 & Less than 5 Years & 4.9 \\
\hline
\end{tabular}

Table 2. Investigation on the Necessity of Establishing New Standards of MEWS in Cardiovascular Medicine.

\begin{tabular}{lll}
\hline Options & Amount & Proportion /\% \\
\hline Very necessary & 64 & 30.92 \\
Necessary & 89 & 43.00 \\
Basically necessary & 34 & 11.18 \\
No necessary & 11 & 5.31 \\
Not necessary at all & 9 & 4.35 \\
\hline
\end{tabular}

\subsection{The Current Application of MEWS in Cardiovascular Medicine and the Necessity of Establishing a New Standard}

According to the survey, $45.8 \%$ of the experts reported that MEWS scoring had not been applied in the department of cardiology, and $54.2 \%$ of the experts said that MEWS scoring had been uesd in clinical practice. Necessity was classified as "very necessary, necessary, basically necessary, not necessary, and not necessary" according to the LIKE-5 grading method. The surveyed experts rated the necessity of establishing a new MEWS standard in cardiovascular medicine. The results showed that $73.9 \%$ of experts believed that it is necessary or very necessary to establish a modified MEWS in cardiovascular medicine. The specific results are shown in Table 2.

\subsection{Reliability Test and Validity Test of Cardiovascular MEWS Index Questionnaire}

\subsubsection{Survey Results}

Assign points to each option: choose "Very necessary" to 
get 5 points, choose "Necessary" to get 4 points, choose "Basically necessary" to get 3 points, choose "No necessary" to get 2 points, and choose "Not necessary at all" to get 1 point.
The statistical results of the Cardiovascular Disease Corrected MEWS Index Survey Scale are shown in Table 3.

Table 3. Significance of survey indicators scoring results.

\begin{tabular}{lllllll}
\hline Index & $\mathbf{1}$ & $\mathbf{2}$ & $\mathbf{3}$ & $\mathbf{4}$ & The average score \\
\hline Systolic blood pressure & 1 & 2 & 9 & 18 & 177 \\
Diastolic blood pressure & 2 & 1 & 10 & 8 & 23 & 19 \\
Breathe & 2 & 5 & 6 & 32 & 169 \\
Heart rate & 2 & 4 & 1 & 33 & 163 \\
Arrhythmia & 0 & 0 & 6 & 39 & 173 \\
Awareness & 1 & 2 & 8 & 26 & 159 \\
Chest pain & 2 & 3 & 5 & 29 & 168 \\
Blood oxygen saturation & 0 & 2 & 4.70 & 4.71 \\
\hline
\end{tabular}

\subsubsection{Reliability and Validity Test}

The reliability test is performed by Cronbach's $\alpha$ coefficient method. The research results show that the reliability coefficient of this questionnaire is 0.989 , and the result is greater than 0.8 , indicating that the reliability and validity of the questionnaire is good. Respectively deleting each item in the questionnaire, the total reliability of the questionnaire did not increase significantly, indicating that all the indicators in the questionnaire can be retained.

\subsubsection{Validity Test}

The validity test of the questionnaire adopts $\mathrm{KMO}$ value and Bartlett's sphere test. The research results show that the $\mathrm{KMO}$ value of this questionnaire is 0.916 , which is greater than 0.8; the Chi-Square value of Bartlett's sphere test is 3670.769 (degrees of freedom is 28), reaching a significant level $(\mathrm{P}<0.000)$, indicating the validity of this questionnaire better. The specific results are shown in Table 5 .
Table 4. Measurement item Cronbach's $\alpha$ coefficient test.

\begin{tabular}{ll}
\hline Index & Cronbach's $\boldsymbol{\alpha}$ coefficient \\
\hline Systolic blood pressure & 0.982 \\
Diastolic blood pressure & 0.982 \\
Breathe & 0.982 \\
Heart rate & 0.982 \\
Arrhythmia & 0.989 \\
Awareness & 0.984 \\
Chest pain & 0.981 \\
Blood oxygen saturation & 0.983 \\
Scale & 0.989 \\
\hline
\end{tabular}

Table 5. KMO value and Bartlett's sphericity test.

\begin{tabular}{lll}
\hline KMO value and Bartlett's sphericity test & \\
\hline Kaiser-Meyer-OlKin metric & Sampling appropriate quantity & 0.916 \\
& Approximate chi-square & 3760.769 \\
Bartlett's sphericity test & Df & 28 \\
& Sig. & 0.000 \\
\hline
\end{tabular}

Table 6. Adjusted MEWS rating scale.

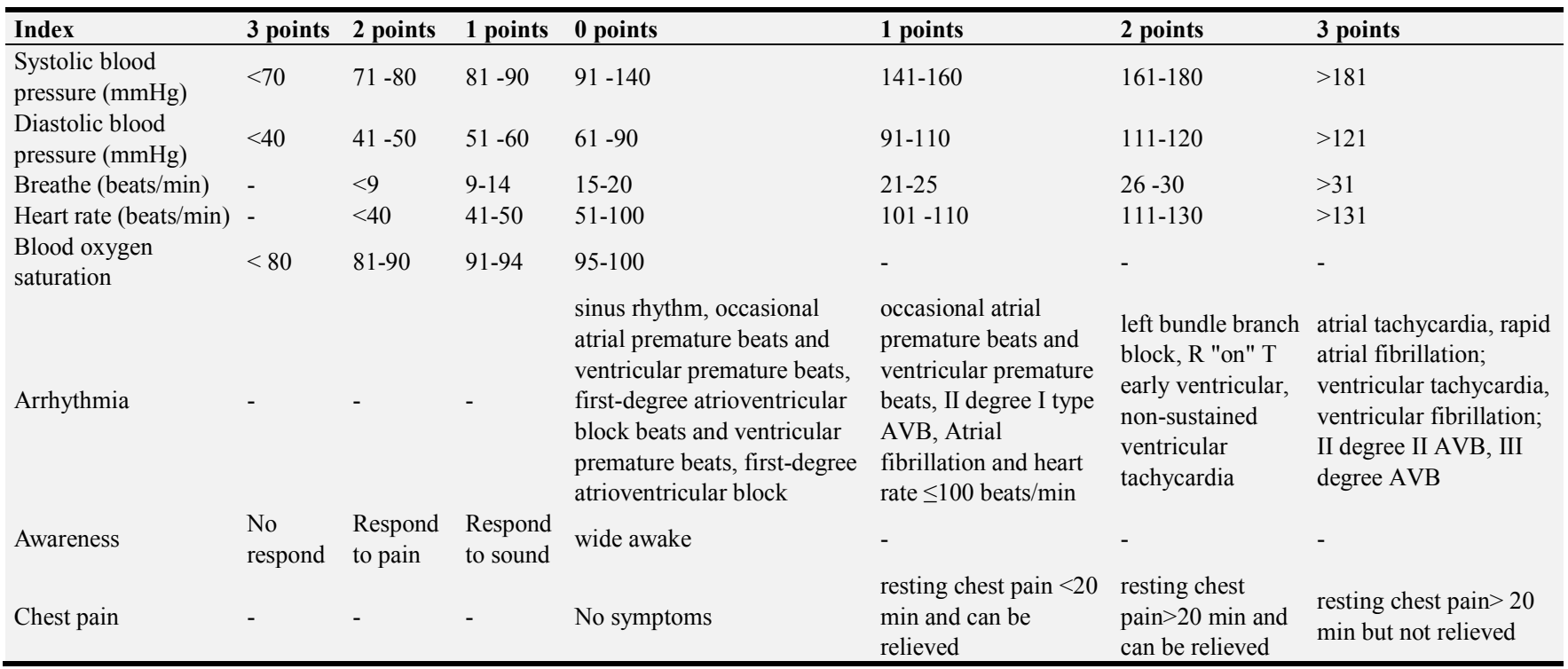

\subsection{Formed an Information-Based Early Warning Monitoring Program for Postoperative Patients Based on the Corrected MEWS Score}

The corrected Mews score is shown in Table 6. According to the scores, the first-level nursing and critically ill patients are scored for cardiovascular medical care early warning, the early warning levels are divided according to the scores, and corresponding measures are taken. In the low-risk group (0-3 points), the patients should be inspected at least every 1 hour 
and repeat the score; in the intermediate-risk group (4-6 points), the doctor on duty and the nursing team leader should be notified, and the patients will be inspected every 1 hour, and the score will be repeated to establish Two venous channels; high-risk group (7-10 points) or a single item is divided into 3 points, the doctor on duty should be notified immediately for preliminary medical treatment. For example, increase the oxygen concentration, evaluate the intake and output, keep the fluid unobstructed, and maintain the airway unobstructed. Re-evaluation will be carried out every 30 minutes, and the head nurse will be notified at the same time, and additional nursing staff will be notified; for extremely high-risk groups ( $\geq 11$ points), infrom the doctor on duty and the nursing team leader, Prepare rescue medicines and items, re-scoring every 10-15 minutes, the superior physician should immediately participate in the rescue of the patient.

\section{Discussion}

The corrected MEWS score for cardiovascular disease can help nurses quickly and accurately identify the patient's condition, and provide a standardized tool for communication between doctors and nurses [9]. A large number of studies [10-15] proposed that cardiovascular diseases need to build an early warning scoring system suitable for disease characteristics. It can be seen from the results of this survey that although the MEWS score has a certain degree of application in cardiovascular medicine, it has not been widely popularized and promoted, indicating that the application of MEWS score in cardiovascular medicine has room for further improvement in the future. $73.9 \%$ of the experts indicated that it is necessary to establish a new MEWS scoring standard for cardiovascular internal medicine, and these experts are nursing experts with many years of clinical experience from tertiary A hospitals, indicating that the construction of a new scoring standard has reality among nursing workers Demand and a high degree of recognition.

Acceptability of cardiovascular disease early warning score scale. In the field of cardiovascular disease, the severity of patients' needs should be objectively and quantitatively assessed, and the changes of patients' conditions could be identified early to achieve effective risk management. Appropriate treatment measures and nursing interventions should be taken in time to improve the prognosis of patients. According to the characteristics of specialized diseases, we modified the scoring items based on the five conventional physiological indicators of heart rate, systolic blood pressure, respiratory rate, body temperature and consciousness based on the traditional MEWS scoring, deleted body temperature items, and added diastolic blood pressure, chest pain, and arrhythmia And blood oxygen saturation four observation items indicators. After reliability and validity tests, the results of reliability and validity are good, indicating that this score sheet is highly reliable and can truly reflect the severity of cardiovascular disease. The entries in this scoring table are concise, and the scoring method is simple and easy to operate. Although the items in the scale still lack some characteristic indicators for the diagnosis of cardiovascular diseases, such as troponin, myocardial enzymes and other laboratory test indicators, the length of the table is simplified to ensure the timeliness of the evaluation of the scale and ensure Nursing staff can quickly evaluate in clinical work, saving the time of treating patients.

\section{Conclusion}

This study modified the scoring items of the traditional MEWS scoring scale using questionnaire survey, combined with the characteristics of cardiovascular system diseases and clinical needs. The length of the table is simplified to ensure the timeliness of the evaluation of the scale and ensure Nursing staff can quickly evaluate in clinical work, saving the time of treating patients. It explored the feasibility and necessity of establishing an early warning scoring scale in line with the features of cardiovascular quasi - feasible, and preliminarily formed a disease assessment system for patients in cardiovascular specialty.

\section{Funding}

2019 Zhuhai City Medical and Health Technology Plan Project;

Project Name: Construction and Application of Early Warning Care Work Model for Cardiovascular Diseases Based on the Corrected MEWS Scoring System;

Project Number: 20191207A010021; Project Leader: Wu Juxian.

\section{References}

[1] Man Y, Qiaofang Y, Binbin Y. Construction and application of the early warning scoring scale for cardiovascular diseases [J]. Chinese Journal of Modern Nursing, 2018, 24 (21): 2525-2528.

[2] Huang Z, Dong W, Duan H. A probabilistic topic model for clinical risk stratification from electronic health records $[\mathrm{J}]$. Journal of Biomedical Informatics, 2015 (58): 28-36.

[3] Shaikh M A, Punshi A, Talreja M L, et al. Comparison of within 7 Day All-Cause Mortality among HDU Patients with Modified Early Warning Score of $\geq 5$ with those with Score of $<$ 5 [J]. Pakistan journal of medical sciences, 2021, 37 (2): 515-519.

[4] Jiang X, Jiang P, Mao Y. Performance of Modified Early Warning Score (MEWS) and Circulation, Respiration, Abdomen, Motor, and Speech (CRAMS) score in trauma severity and in-hospital mortality prediction in multiple trauma patients: a comparison study [J]. PeerJ, 2019, 7: e7227.

[5] Mitsunaga T, Hasegawa I, Uzura M, et al. Comparison of the National Early Warning Score (NEWS) and the Modified Early Warning Score (MEWS) for predicting admission and in-hospital mortality in elderly patients in the pre-hospital setting and in the emergency department [J]. PeerJ, 2019, 7: e6947.

[6] Yu Z, Xu F, Chen D. Predictive value of Modified Early Warning Score (MEWS) and Revised Trauma Score (RTS) for the short-term prognosis of emergency trauma patients: a retrospective study [J]. BMJ Open, 2021, 11 (3): e41882. 
[7] Devoe B, Roth A, Maurer G, et al. Correlation of the predictive ability of early warning metrics and mortality for cardiac arrest patients receiving in-hospital Advanced Cardiovascular Life Support [J]. Heart \& Lung the Journal of Critical Care, 2016: 497-502.

[8] Young K W, Jung S Y, Mi L J, et al. Modified Early Warning Score Changes Prior to Cardiac Arrest in General Wards [J]. Plos One, 2015, 10 (6): e0130523.

[9] Qingwei Z, Qiaofang Y, Man Y. Clinical application and thinking of risk assessment and early warning tools in cardiovascular diseases [J]. Journal of Nursing, 2019, 26 (16): 34-37.

[10] Yanming L, Ruili H, Xiaoming Z, et al. Establishment and study of prognostic evaluation model for patients with acute ST-segment elevation myocardial infarction after PCI [J]. Chongqing Medicine. 2019 (01): 71-75.

[11] Yonghong W. The application of early warning scoring system in emergency PCI treatment of acute myocardial infarction and the influence of patients' heart function [J]. International Journal of Nursing, 2021, 40 (05): 930-933.
[12] Zhihua $H$. The effect of the improved early warning scoring system on the pre-examination and triage of patients with emergency chest pain [J]. Electronic Journal of Integrated Traditional Chinese and Western Medicine Cardiovascular Diseases, 2019, 7 (011): 182.

[13] Hong L, Tingting M, Yan M. Study on the trend of early warning scores in patients with acute coronary syndromes 24 hours before cardiac arrest [J]. Chinese Journal of Nursing, 2019, 54 (01): 14-18.

[14] Liling $\mathrm{H}$. The impact of early warning risk management on the occurrence of adverse cardiovascular events in patients with coronary heart disease [J]. Tibet Medicine, 2020, 41 (01): 102-104.

[15] Bo Y, Fangyan L, Xiao L, et al. A comparative study of NT-proBNP and risk score for early warning of major adverse cardiovascular events in the hospital for emergency ACS patients [J]. Chinese Journal of Emergency Medicine, 2017, 26 (09): 1071-1075. 